\title{
Contrast enhanced ultrasound of a hepatic soft tissue angiosarcoma metastasis. Case report.
}

\author{
Maximilian Rauch, Hans H. Schild, Holger Strunk
}

Department of Radiology, University Hospital Bonn, Germany

\begin{abstract}
Diagnostic imaging of angiosarcoma and angiosarcoma metastasis has been described as confusing and challenging. We present a rare case of hepatic soft tissue angiosarcoma metastasis, which was diagnosed by contrast enhanced ultrasound (CEUS). The case further exemplifies the ability of CEUS to solve discrepancies between other imaging modalities.

Keywords: hemangiosarcoma, liver, ultrasound, microbubbles
\end{abstract}

\section{Introduction}

Soft tissue angiosarcoma is a rare, aggressive tumor with a poor prognosis. It frequently metastasizes to the lung, bone, lymph nodes and less often to the liver. To the best of our knowledge, no case of contrast enhanced ultrasound (CEUS) regarding a hepatic soft tissue angiosarcoma metastasis has been reported. We refer to the case of a 27 year old woman with a history of soft tissue angiosarcoma who presented with a hip and liver lesion.

\section{Case report}

A 27 year old foreign female patient with a history of soft tissue angiosarcoma was referred to the orthopedic department of our institution due to right-sided hip pain for the last 6 months which aggravated during the

Received 25.06.2014 Accepted 30.06.2014 Med Ultrason

2014, Vol. 16, No 3, 271-273

Corresponding author: Dr. Maximilian Rauch

University Hospital Bonn

Department of Radiology

Sigmund-Freud-Strasse 25

D-53127 Bonn, Germany

Phone: +49 22828711818

Fax: +49228287 11166

E-mail: maximilian.rauch@ukb.uni-bonn.de last weeks. She was diagnosed with a soft tissue angiosarcoma of the left anterior thoracic wall (T2b N0 M0) three years before, which was completely removed surgically (R0). She received no further adjuvant therapy at that time, but was checked regularly by radiologic exams abroad, which reportedly showed no abnormalities. The patient did not have a history of radiotherapy, environmental carcinogen exposure or other relevant diseases. Clinical examination showed limited right leg mobility due to strong right sided hip pain. Further clinical examination and laboratory tests were normal. The patient did not experience weight loss, fever or fatigue.

Computed tomography (CT) and subsequent positron emission tomography (PET)-CT showed an infiltrating, flourodeoxyglucose (FDG)-avid mass in the right acetabulum, iliac wing and superior pubic ramus (fig 1a), which proved to be an angiosarcoma metastasis upon biopsy. In addition, a solitary $5.5 \times 4.0 \times 4.6 \mathrm{~cm}$ peripherally and centrally nodularly enhancing hypodense liver lesion was found (fig 1b). PET-CT revealed no evidence for increased metabolic activity of the liver lesion (fig 1a).

Due non-specific lesion characteristics and the discrepancy of CT and PET-CT findings, differential diagnosis included liver hemangioma and angiosarcoma metastasis. Accordingly, CEUS was performed for further lesion characterization using a Logiq E9 unit (GE Healthcare) and a $3.5-5.0 \mathrm{MHz}$ curved array transducer. 
The preceding non-enhanced B-mode scan showed an inhomogenous mass with sparse internal and peripheral flow signals on Doppler imaging (fig 2). The imaging mode was changed to CEUS with a low mechanical index (MI) of $<0.1$ and $1.2 \mathrm{ml}$ sulfur hexafluoride microbubble contrast medium (SonoVue, Bracco) was injected intravenously followed by a $10 \mathrm{ml}$ saline flush. In the arterial phase, the lesion exhibited sparse peripheral but intense internal nodular enhancement (starting from 17 seconds), which was persistent in the late phase and failed to exhibit washout. Most of the mass did not exhibit contrast enhancement. No progressive centripetal or centrifugal filling was seen in portal venous and late phases (fig 3 ). These findings did not match those reported for typical and atypical liver hemangioma, but were similar to the reported CT and MR characteristics of primary liver angiosarcoma and hepatic metastasis of soft tissue angiosarcoma. We therefore diagnosed hepatic soft tissue angiosarcoma metastasis. Percutaneous biopsy of the liver lesion was refused due to the lack of further consequence after the patient decided for a palliative tumor debulking and hip-replacement prosthesis to preserve mobility.

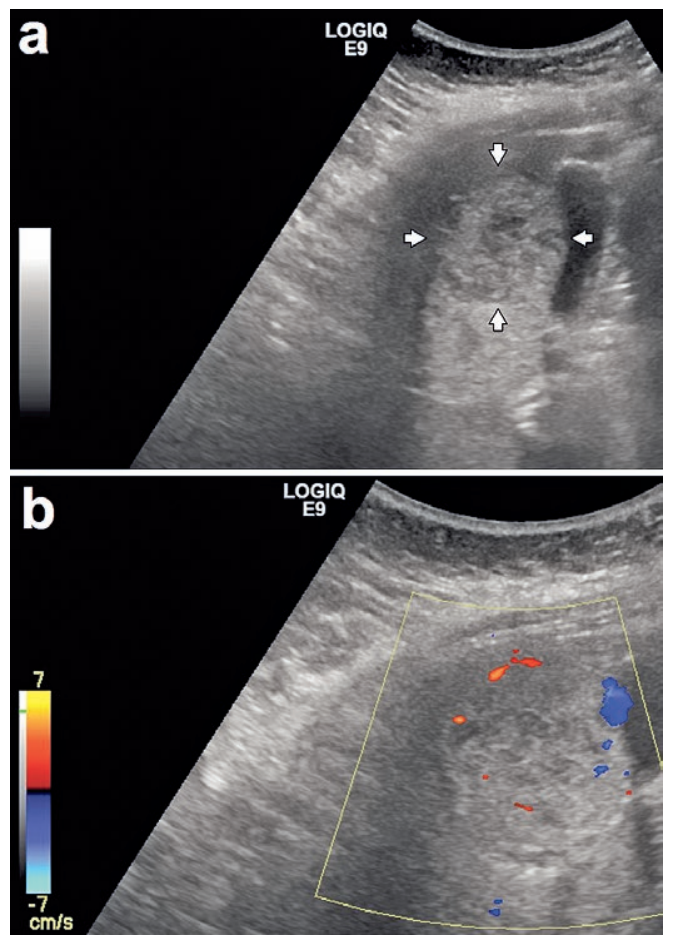

Fig 2. a) B-mode sonography of the liver lesion showing an inhomogenous mass (arrows) with sparse peripheral and internal color Doppler signals (b). chemotherapy (b), and regression after first (c) and second (d) cycle of chemotherapy.

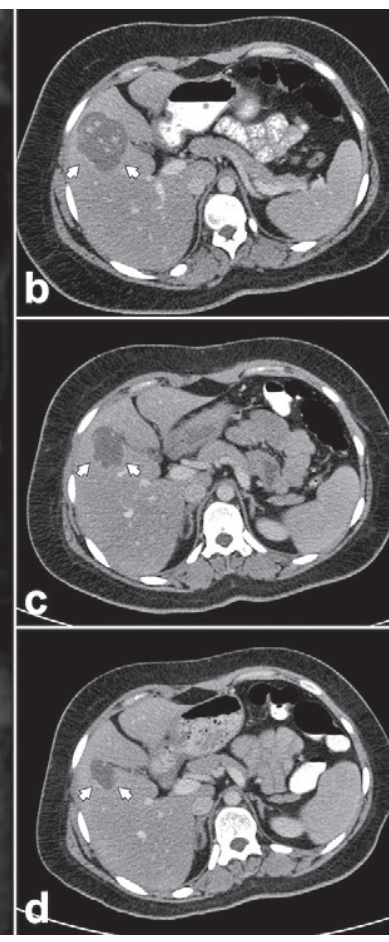

Fig 1. a) Coronal PET (MIP) showing FDG-avid lesion of the right hip (arrows). No increased FDG avidity of the liver le-

Fig 3. Contrast enhanced ultrasound (CEUS) of the liver lesion. Sparse peripheral and intense internal nodular enhancement of the liver lesion in the arterial phase. No progressive centripetal or centrifugal filling is seen in the portal venous and late phase. Most of the tumor mass shows no enhancement. 
After convalescence from hip replacement surgery, a targeted radiotherapy of the hip and a systemic chemotherapy regimen including doxorubicin and ifosfamide was initiated.

Diagnosis was confirmed by clinical course and radiologic follow-up, which showed a considerable regression after the first (fig 1c) and second cycle (fig 1d) of chemotherapy.

\section{Discussion}

Diagnostic imaging of angiosarcoma has been described as challenging, showing unspecific findings, hence potentially being confused with benign vascular lesions $[1,2]$.

Our CT findings depicted a solitary hypodense hepatic lesion with sparse peripheral and intense internal nodular enhancement. These findings were comparable to those described by Tateishi et al, who studied hepatic soft tissue angiosarcoma metastasis. They found various CT-imaging results comparable to findings of primary hepatic angiosarcoma, including hypoattenuating nodules with peripheral areas of enhancement, cystic attenuation nodules with fluid-fluid levels suggestive of hemorrhage or markedly enhancing nodular portions located centrally or peripherally [3].

The liver lesion was not FDG-avid on PET-CT. PET$\mathrm{CT}$ is well established for the staging of primary softtissue sarcomas, whereas its value in the detection of soft-tissue sarcoma metastases remains unclear due to wide-ranging reported sensitivities and specificities [4].

Our non-enhanced B-mode and Doppler flow imaging results were comparable to those described for primary hepatic angiosarcoma exhibiting non-specific variable degrees of echogenicity with sparse peripheral and internal flow signals $[1,2]$.

CEUS is established for the characterization of liver lesions and allows real-time imaging of organ and lesion perfusion, thus exhibiting comparable results to $\mathrm{CT}$ and magnetic resonance imaging (MRI) studies in focal liver lesion characterization [5]. It is frequently employed in clinical practice to investigate lesions or pseudolesions indeterminate at other imaging modalities. CEUS has further been shown to be accurate in the diagnosis of typical hepatic hemangioma, the most important differential diagnosis in our reported case. Imaging hallmarks of hemangioma include a mass with peripheral nodular enhacement, progressive centripetal filling and persistent enhancement in later phases. Filling may be rapid ('flash- filling') in small lesions or incomplete in larger hemangiomas due to thrombosis, hemorrhage or fibrosis.

On CT and CEUS we found sparse peripheral enhancement and central non-enhancing areas, untypical for hemangioma but rather comparable to findings reported for primary liver angiosarcoma. This has been shown to exhibit different enhancing patterns with peripheral irregular enhancement and lack of progressive centripetal filling being the most common observed features [1,2]. Central non-enhancement of primary liver angiosarcomas has been attributed to central necrosis, thrombosis, hemorrhage or fibrosis, whereas areas of peripheral enhancement correspond to foci of proliferating neoplastic cells. However, Wang et al. characterized primary liver angiosarcomas using CEUS and unexpectedly found vital tumor tissue in corresponding non-enhancing central areas histologically [1]. It was therefore reasoned that, comparable to hemangioma, non-enhancement may be related to low blood flow velocities in intratumoral vascular spaces.

\section{Conclusion}

In the reported case, CEUS of hepatic angiosarcoma metastasis showed findings comparable to those described for $\mathrm{CT}$ and MR imaging of primary liver angiosarcoma and hepatic soft tissue angiosarcoma metastasis. CEUS is an excellent tool for characterization of liver lesions, especially in indeterminate cases. Nevertheless, it should be interpreted within the particular patient's clinical context and course.

\section{References}

1. Wang L, Lv K, Chang XY, et al. Contrast-enhanced ultrasound study of primary hepatic angiosarcoma: a pitfall of non-enhancement. Eur J Radiol 2012; 81: 2054-2059.

2. Trojan J, Hammerstingl R, Engels K, Schneider AR, Zeuzem S, Dietrich CF. Contrast-enhanced ultrasound in the diagnosis of malignant mesenchymal liver tumors. J Clin Ultrasound 2010; 38: 227-231.

3. Tateishi U, Hasegawa T, Muramatsu Y, Moriyama N. Hepatic metastases of soft tissue angiosarcoma: CT and MR imaging findings. Abdom Imaging 2003; 28: 660-664.

4. Roberge D, Vakilian S, Alabed YZ, Turcotte RE, Freeman CR, Hickeson M. FDG PET/CT in Initial Staging of Adult Soft-Tissue Sarcoma. Sarcoma 2012; 2012: 960194.

5. Sporea I, Sirli R. Is Contrast Enhanced Ultrasound (CEUS) ready for use in daily practice for evaluation of focal liver lesions? Med Ultrason 2014; 16: 37-40. 